Journal of Engineering and Applied Sciences 14 (11): 3588-3594, 2019

ISSN: 1816-949X

(C) Medwell Journals, 2019

\title{
Assessment of Smoke Control Performance via. Fire Simulations and Hot Smoke Tests
}

\author{
${ }^{1}$ Dongwoo Song, ${ }^{2}$ Sukyung Lee and ${ }^{2}$ Keumsook Hwang \\ ${ }^{1}$ Department of Fire Protection Safety Engineering, Oklahoma State University, Stillwater, USA \\ ${ }^{2}$ Department of Safety Engineering, Seoul National University of Science and Technology, \\ Seoul, South Korea
}

\begin{abstract}
In Atria and other large spaces, smoke from fires can flow freely, leading to unusual patterns of smoke flow. When smoke control systems are designed in Korea, the designer can propose the use of Performance-Based Design (PBD) methods. In the present study, we perform Computational Fluid Dynamics (CFD) modeling and Hot Smoke Tests (HSTs) to implement PBD for an atrium. This study introduces an optimal smoke control system to a target site by comparing the properties of CFD modeling and onsite HSTs in three design scenarios using a forced air supply/exhaust, natural air supply and natural exhaust via. smoke exhaust windows. HSTs and a fire dynamics simulator are applied to each scenario and the results are used to identify variables affecting smoke flow. We distinguish the separate effects of forced ventilation, smoke exhaust window position and natural air supply on smoke flow. These methods can be used as tools to determine the operating conditions for smoke control systems, the effects of supply/exhaust vent positioning on smoke control and building administration measures for Atria of various shapes.
\end{abstract}

Key words: Atria, performance-based design, large space, smoke management system, smoke detection, fire dynamics simulator, smoke flow

\section{INTRODUCTION}

In Atria with at least two open floors or other long high-ceilinged spaces within buildings smoke can flow freely during a fire. In addition, unusual patterns of smoke flow develop due to indoor temperature differences, an outside temperature gradient when doors are opened and air currents from air conditioning units. In such spaces, fire simulation analysis can be used to test the performance of smoke control systems. Fire simulation is a useful tool for evaluating the performance of smoke control systems at the design stage. However, it is impossible to test all aspects of large spaces like Atria. Webb (2006) performed HSTs in a cinema and airport concourse and compared the results with FDS modeling. $\mathrm{He}$ found that the two methods exhibited similar temperature and smoke density but differences in air speed that could be explained by leakage, air supply vent placement and changes in airflow after turning on smoke extractor fans. Chow et al. (2009) compared the results of HSTs and a CFD tool and found that the height of the smoke layer was similar across the two methods but that they differed in temperature predictions. Hence, they proposed that when CFD is used in design, its results should be verified experimentally.
No separate regulations exist for smoke control systems in Atria. PBD methods have been introduced when designing smoke control systems, so that, designers can propose smoke control methods for the relevant space. This system provides PBD as an engineering approach to fire protection that is designed according to fire scenario analysis and quantitative assessment using accepted engineering tools, methodologies and performance criteria presented in the SFPE handbook. In Korea, the Firefighting Technology Committee approves buildings on the condition that onsite HSTs are performed to compensate for the shortcomings of CFD analysis. HSTs are suited to realistic smoke flow analysis because they reflect all the spatial characteristics of the structure after construction is completed. In Australia, HSTs are generally carried out in accordance with AS 4391-1999, a standard that provides a method for assessing the sequential operation of a building's smoke management system (Standard, 1999). In Hong Kong, HSTs are carried out where specified by FSD Circular Letter No. 2/2002, Australian Standard AS 4391-1999 or approved international standards and practices to the satisfaction of the FSD and a PBSE. In a study evaluating the performance of mechanical smoke

Corresponding Author: Keumsook Hwang, HIMEC Fire and Life Safety, World Meridian Biz Center 8F, Seoul National University of Science and Technology, Yangsan-ro, Yeongdeungpo-gu, 150-103 Seoul, Korea 
exhaust systems (Chow et al., 2005) carried out HSTs in a large shopping complex, aiming to demonstrate that its smoke exhaust system's operation satisfied the smoke layer criterion. In this study, burning methylated spirits were used to create buoyant smoke during the HST. The evaluation was made using visual observation and vertical temperature profile measurement by thermocouples. As is most typical, the smoke layer interface was observed visually through indication marks and the vertical temperature profiles were measured in accordance with AS 4391-1999 (Standard, 1999). In Korea, however, HSTs can only be visually observed without measuring the temperature profile. Visual observation is sufficient for judging whether fire protection equipment and systems are working but it is insufficient for quantitatively judging the reduction in the smoke layer. The performance of HSTs using burners with liquid fire and thermocouples as per Australian standards is difficult in Korea due to equipment damage in buildings and unsafe experiments. Therefore, in this study, we used heaters for rising smoke and detectors installed in the building to overcome the deficiencies of visual observation.

In this study, the performance and operation of the smoke control system can be suggested by using detectors installed in the building. The Atrium space investigated in the present study used a PBD approach verified by FDS and HSTs. Herein, we do not aim to validate CFD but rather to compare the properties of CFD modeling results and onsite HSTs in different scenarios and use this information to introduce an optimal smoke control system to the site.

\section{MATERIALS AND METHODS}

Target building and smoke control scenarios: The target building is used by the public and requires rapid restoration in the event of an emergency. Therefore, the aims of $\mathrm{PBD}$ are to protect the lives of users and to ensure continuity of business. The target for PBD is an atrium space with three open floors. The design scenarios were based on the highest floor within the open atrium space without a fire plan. The floor area of the Atrium is broadest on level 2, at $8780 \mathrm{~m}^{2}$ and was $1590 \mathrm{~m}^{2}$ on level 4 . The Atrium ceiling is $31.3 \mathrm{~m}$ at its highest point and $22.8 \mathrm{~m}$ at its lowest point. The area that, we aim to maintain as the clear layer was $1.8 \mathrm{~m}$ from the floor on level 4 and $14.9 \mathrm{~m}$ from the floor on level 2. In other words, a clear layer needed to be maintained at a height where occupants on the top floor would be able to escape without being affected by smoke $(1.8 \mathrm{~m}$ from the
Table 1: Methods of ventilation by scenario

\begin{tabular}{llll}
\hline Type of ventilation & Scenario 1 & Scenario 2 & Scenario 3 \\
\hline Forced supply & Yes & No & No \\
Forced exhaust & Yes & No & Yes \\
Natural supply & No & No & Yes \\
$\begin{array}{l}\text { Natural exhaust } \\
\text { (smoke exhaust windows) }\end{array}$ & Yes & Yes & No \\
\hline
\end{tabular}

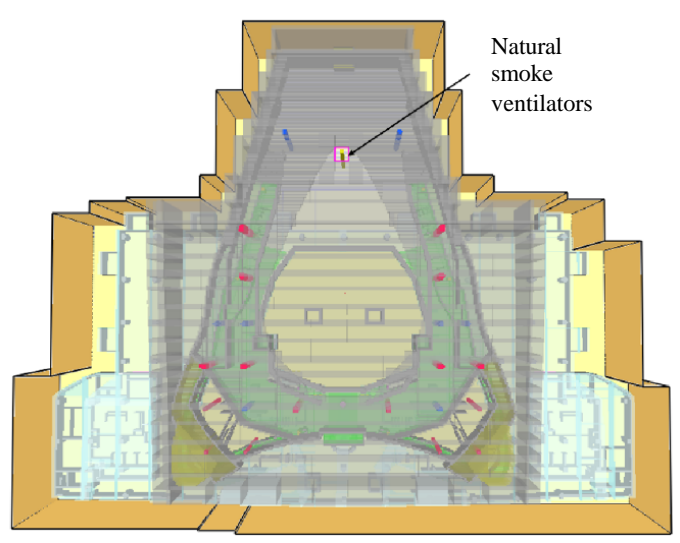

Fig. 1: The appearance of the simulation model

floor (MGL., 2017). In addition, smoke must be rapidly eliminated, so that, the building can be used again. Achieving the above goals requires proper smoke control equipment operation. In this study, to analyze the change in smoke concentration over time, we examine smoke extraction performance in several scenarios. The smoke control equipment that can be used at the target site includes a forced air supply and exhaust, natural air supply and natural air exhaust via. smoke exhaust windows. Hence, we test the three smoke control equipment scenarios shown in Table 1.

The first scenario is a mechanical supply and exhaust system with smoke exhaust windows located at the front of the highest point of the atrium (in accordance with limiting architectural conditions) as shown in Fig. 1, that are manually opened $5 \mathrm{~min}$ after firefighting services are mobilized. The area of the smoke exhaust windows is $10.24 \mathrm{~m}^{2}$. As shown in Fig. 2, the supply vents are installed on the lower level and the exhaust vents are installed on the upper level. The exhaust flow rate is set at $240,000 \mathrm{CMH}$ in accordance with the atrium smoke removal capacity criteria presented in NFPA 92 and 101 (NFPA., 2010a, b, 2015). The supply flow rate is also set at $240,000 \mathrm{CMH}$ as per the Korean Fire Services Act (NFA., 2017). The second scenario is a natural smoke control system using only smoke exhaust windows. This scenario accounts for the natural way in which smoke fills large spaces. The third scenario consists of opening ten 
external doors directly communicating with the atrium and a mechanical exhaust system utilizing 12 vents installed at the sides of the topmost ceiling.

Hot smoke tests: We performed HSTs to analyze smoke flow as reflected in the spatial properties of the target building after the completion of construction. Several studies and standards have been published addressing HSTs. Chow (2009) conducted an HST in an irregularly

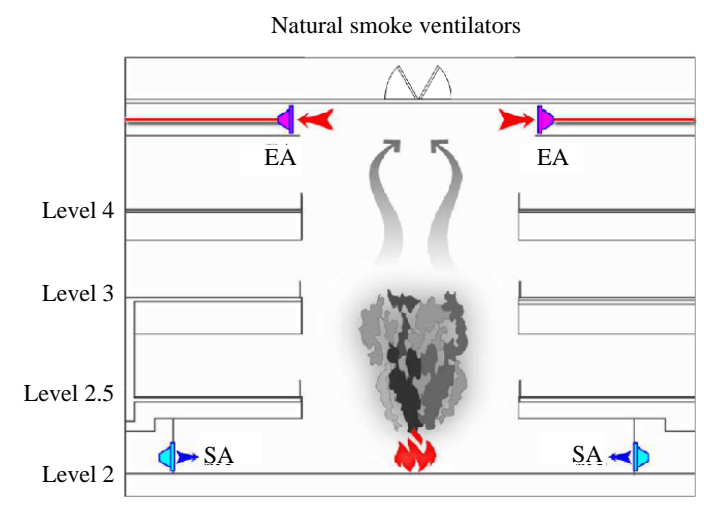

Fig. 2: Mechanical ventilation system and smoke exhaust windows shaped building with a space over $12 \mathrm{~m}$ in height in Hong Kong and reported that while the results are usually analyzed by visual inspection in atria and other large spaces, irregular smoke movements make it difficult to determine the height of the smoke layer with the naked eye because clear smoke layers are not well defined, it is important to measure the vertical temperature distribution. Su et al. (2011) varied the opening of upper and lower-level doors installed in an apartment staircase in Taiwan for ventilation and tested their control of the smokestack effect according to the position of the open doors. In Australia where AS 4391-1999 is adhered to (Standard, 1999), there is discussion about how to improve HSTs. AS 4391-1999 only involves experiments for a single scenario the most ideal state, making corrections by scaling the thermal capacity in a state of no airflow and emphasizes that HSTs cannot describe the actual amount of smoke produced.

While performing HSTs in this study, we made observations with the naked eye and through video analysis. Ten camcorders were used as recording equipment as well as anemometers to measure the airflow velocity at the external doors and analog spotmeters (photoelectric smoke detectors) installed on site for quantitative analysis of smoke concentration by location (Fig. 3 and 4). The programming engineer who installed

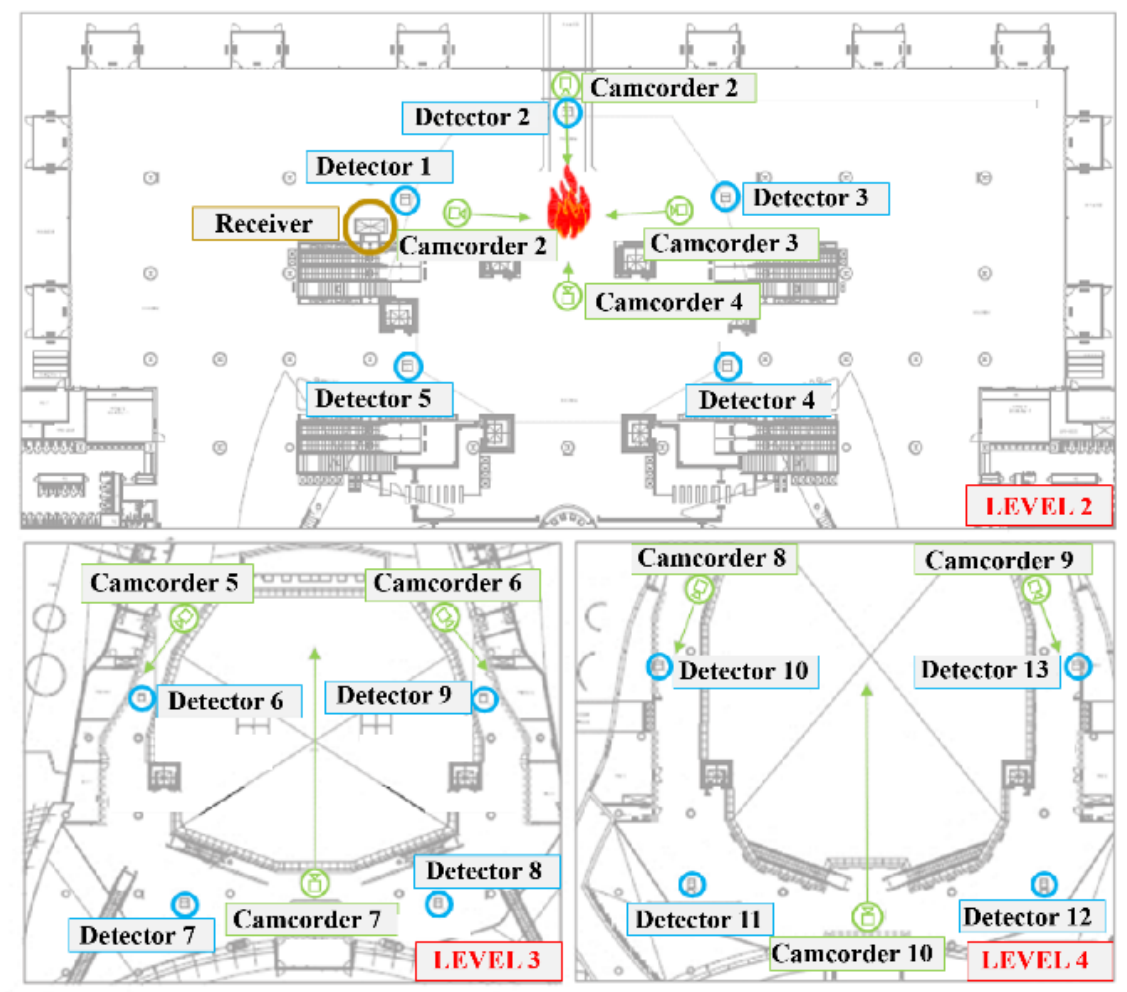

Fig. 3: Equipment locations 


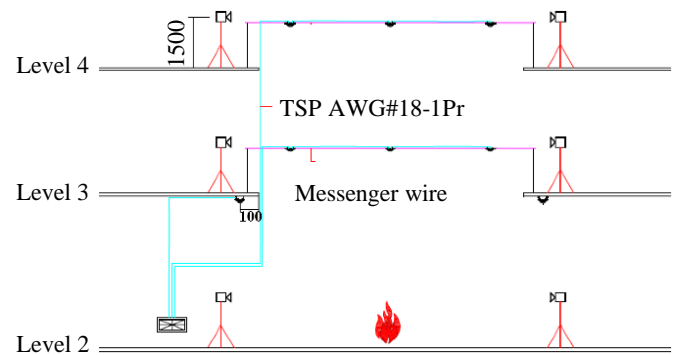

Fig. 4: Floor-by-floor depiction of equipment

the detectors measured the signals from the onsite detectors at intervals of $1 \mathrm{~min}$ to fit the test scenarios. The role of the detectors was not to check whether the fire alarm was triggered but to measure smoke detection at each location for a better understanding of the smoke flow. The atrium is used as train station concourse, given the evacuation time limit of $6 \mathrm{~min}$ required by domestic and international standards, we set the experiment duration to a safe minimum of $10 \mathrm{~min}$ or 1.5 times the evacuation time limit (in accordance with the operating time for smoke control equipment in NFPA 92) (MLIT., 2015; NFPA., 2010a, b).

The smoke generation point was set to the center of the Atrium. A smoke generator manufactured in accordance with ISO 9001 and heater (operating using liquid petroleum gas) were used for fuel. Since, it is impossible to replicate the actual amount of smoke produced, Anonymous (2015) proposed testing along the same pathways using hot buoyant smoke with several types of heat generating devices. In this study, considering the prevention of damage to equipment in the building and safe experimentation, the rise of smoke with the use of heater appeared to be appropriate. Figure 5 shows a photograph of smoke generation during an HST. Table 2 shows the specifications of the equipment used.

Computational simulation: The CFD fire simulation used FDS Version 6 (McGrattan et al., 2015). FDS was developed by the Building and Fire Research Laboratory at the NIST in the United States, it can predict smoke patterns and concentration within a given space during a fire. CFD cannot recreate a fire exactly but its validity and efficacy have been proven through numerous experiments and verifications. Validation studies of FDS have provided many ideas for using models (McGrattan et al., 2017). Webb (2006) studied airflow velocity and smoke flow in FDS and HSTs, these results suggested that the exhaust and supply vent positions and the unclear leakage volume in and out of various openings in the

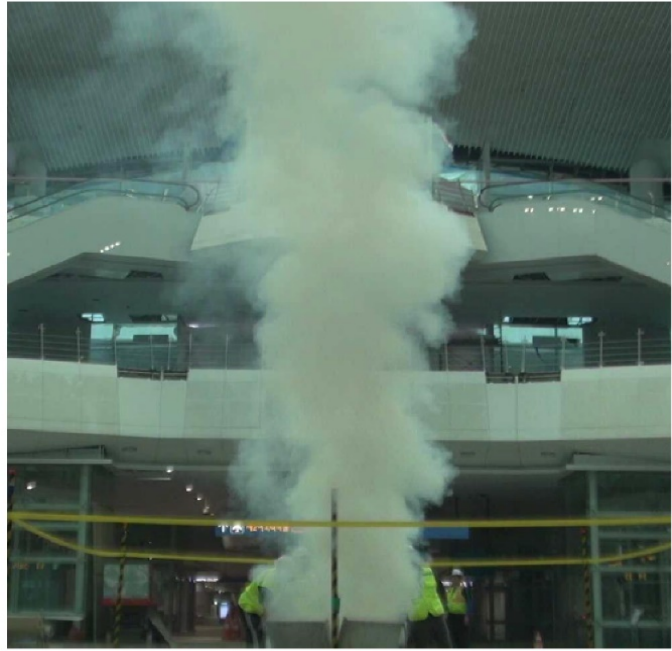

Fig. 5: Photograph of HST smoke generation

Table 2: Specifications for experimental equipment

\begin{tabular}{lc}
\hline Equipment/Specifications & EA \\
\hline Smoke generator & 2 \\
Product: ViCount Compact $5000(2.2 \mathrm{~kW})$ & \\
Smoke output: $0-290\left(\mathrm{~m}^{3} / \mathrm{min} @ 1.0 \mathrm{~m}\right.$ visibility) & 3 \\
Heater & \\
Product: SIP 1706 & \\
Fuel type: Propane & \\
Heat output: $170,607 \mathrm{BTU} / \mathrm{h}(50 \mathrm{~kW})$ & 13 \\
Supply pressure: $1.5 \mathrm{bar}$ & \\
Smoke detector & \\
Product: Photoelectric smoke sensor (Simplex) & 1 \\
Levels of sensitivity: $0.67-12.3 \%$ (obs/m) & \\
Receiver & \\
Product: $4100 \mathrm{ES}$ fire alarm control panel (Simplex) & 1 \\
Anemometer & \\
Product: LCA 6000 (DAVIS) & \\
Velocity range: $0.25-30$ (m/sec) &
\end{tabular}

building cause discrepancies between FDS and HSTs. Thus, in the present study, the locations of exhaust and supply vents were verified onsite and in schematics and were reflected in the model (Anonymous, 2015).

The objective of computational simulation was to design an appropriate fire for the target site and to evaluate smoke performance in several scenarios. To ascertain the change in smoke concentration at each location, we inspected the concentration measured by detectors at the same locations in the HSTs. For the fire, we selected a Christmas tree fire that might occur in an atrium as discussed in the fire scenarios chapter of the SFPE handbook. The fire's heat release rate was $3 \mathrm{MW}$, the fire growth rate was "ultrafast" and the reaction material was Douglas fir wood.

The model lattice which has a major impact on CFD results can be related to the characteristic fire diameter $\mathrm{D}^{*}$ as shown in Eq. 1 and cell size dx. Proposed 
values of ratio $\mathrm{D}^{*} / \mathrm{dx}$ are between 5 and 10 (ASD., 2007; Montes et al., 2009) in this study, we chose a grid size of $200 \mathrm{~mm}$ which makes $\mathrm{D}^{*} / \mathrm{dx}$ close to 10 :

$$
\mathrm{D}^{*}=\left[\frac{\dot{\mathrm{Q}}}{\rho_{0} \times \mathrm{Cp} \times \mathrm{To} \times \sqrt{\mathrm{g}}}\right]^{2 / 5}
$$

In scenario 3, the airflow velocity at the natural supply vents was measured during the HST and used as input data to the FDS. Although, there were differences at each vent, the measurements were in the range of $0.4-1.6 \mathrm{~m} / \mathrm{sec}$, the mean value was used in the simulation. Fig. 1 shows a model of the target space.

\section{RESULTS AND DISCUSSION}

In the HST for scenario 1, smoke was detected at all four detectors installed on level 4 (Detectors 10,11, 12 and 13) and at the two detectors installed on the left and right sides of level 3 (Detectors 6 and 7) (Fig. 6a). During the HST, the smoke concentration at each measurement point became steadier over time, indicating that the smoke exhaust performance was stable. In the FDS, a low

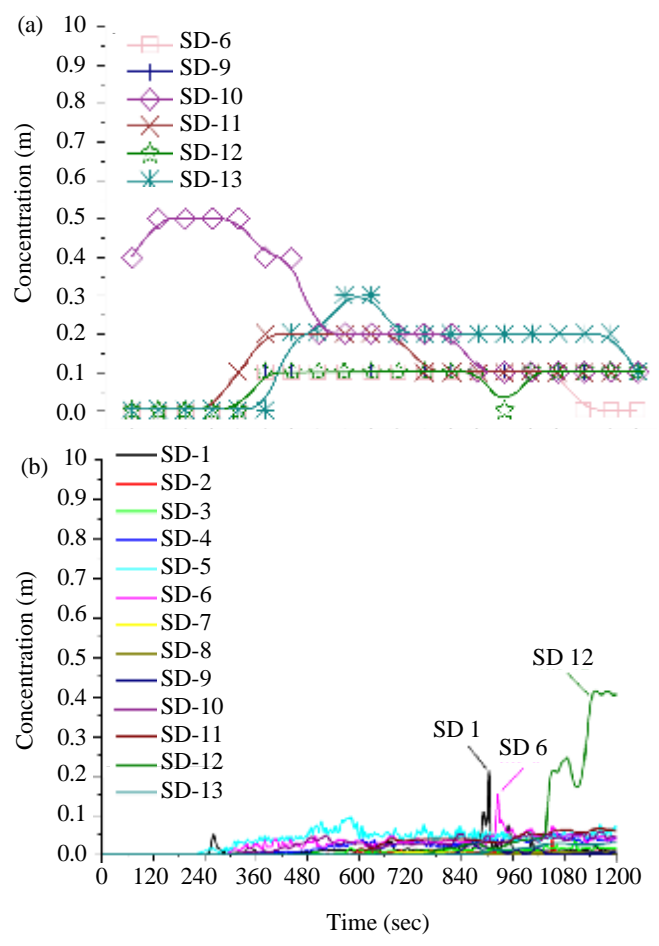

Fig. 6: Scenario 1: a) HST, the smoke concentration is measured in by 6 smoke detectors at the upper level and b) FDS results smoke is detected at low concentrations in smoke detectors concentration of smoke was detected at all detectors (Fig. 6b). These results may show that the mechanical forced air supply on the lower level of the atrium caused the smoke to disperse relatively quickly in both the HST and FDS, resulting in smoke detection at several detectors.

In scenario 2 , the smoke rose vertically solely by buoyancy. In the HST, smoke was detected at three of the detectors on level 4 (Detectors 10, 11 and 13); smoke continuously accumulated at detector 13 (Fig. 7a). Meanwhile, smoke movement towards the smoke exhaust windows (Fig. 1) meant that no smoke reached detector 12. In the FDS, smoke was not detected before the smoke layer had accumulated to a certain height, later, the concentration rose steeply (Fig. 7b), indicating that continual smoke extraction was difficult.

HST results for scenario 3 showed positive smoke exhaust performance in which the measured concentrations at each detector became increasingly stable over time. However, the smoke concentration at detector 6 on level 3 increased (Fig. 8a), possibly because of flow velocity differences between the left and
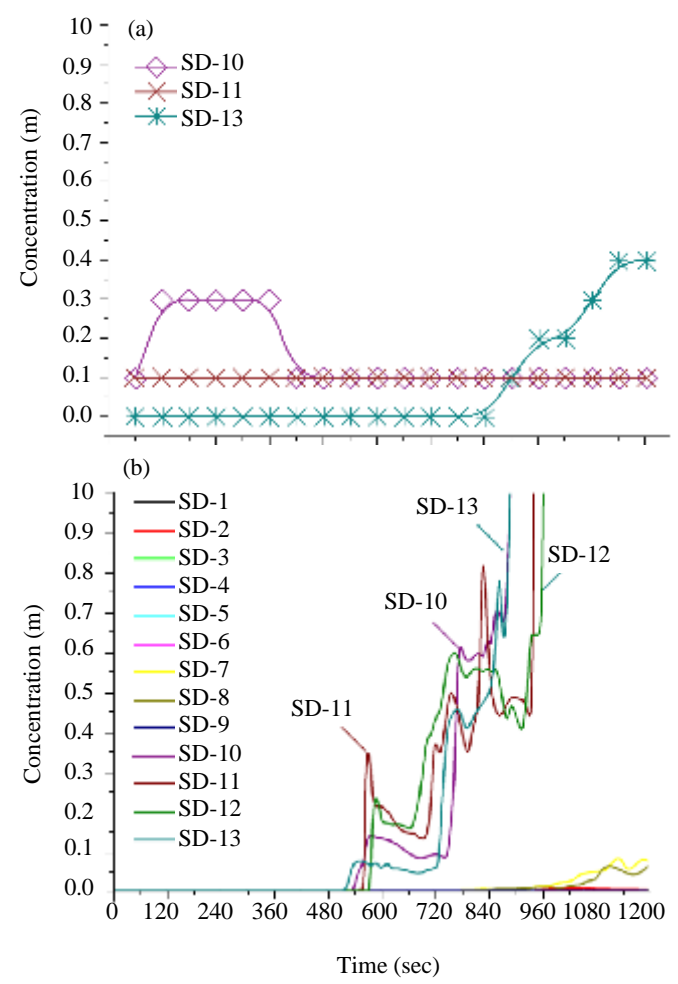

Fig. 7: Scenario 2: a) HST, the concentration is measured in by three sensors at the upper level and the concentration of smoke detector 13 tends to increase and b) FDS results the concentration of smoke detectors at the upper level rises sharply 

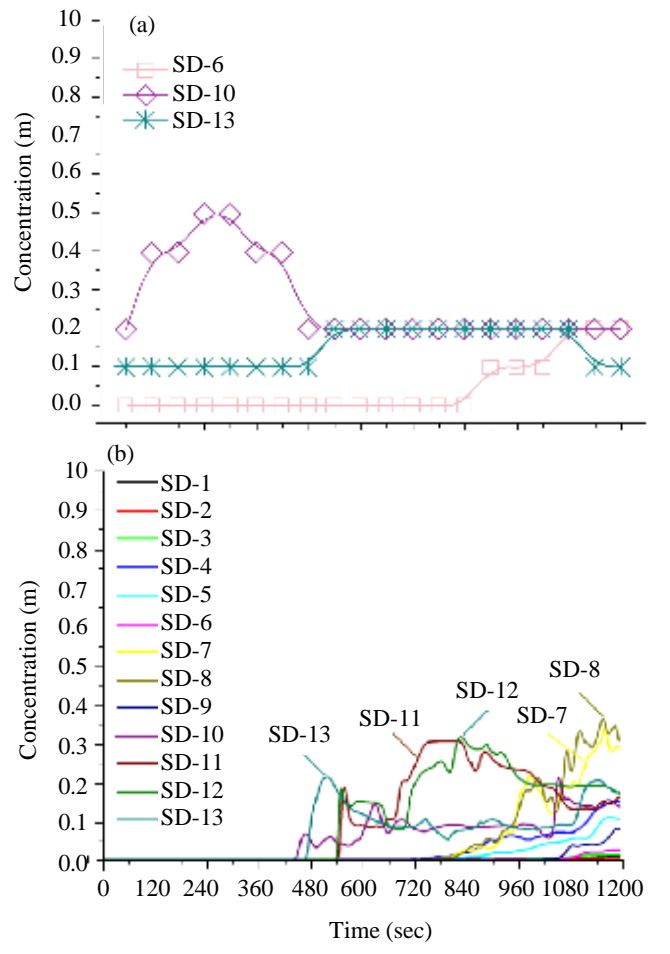

Fig. 8: Scenario 3: a) HST, the concentration is measured in by three sensors at the upper level and the smoke concentration of Detector 6 increased and b) FDS results the smoke concentration is measured in some smoke detectors at the upper level

right vents for the natural air supply in this area. That is the faster air flow velocity lifted the smoke towards the right side before exhaust blew through the mechanical exhaust vents, conversely, the left side did not receive the effects of the supply vents and so the smoke became stagnant. When a natural air supply was implemented, stagnant zones developed due to changes in flow velocity. In the FDS when the amount of smoke generated began to increase significantly, the volume of the natural air supply was relatively low compared to the volume of mechanical smoke exhaust, meaning that the smoke gradually dispersed and was detected at a high concentration at all detectors (Fig. 8b).

In summary, smoke exhaust performance was successful after a time in scenario 1 . However, the high flow rate of the forced mechanical supply vents in the lower part of the atrium resulted in relatively rapid smoke dispersal and caused significant changes in airflow. Scenario 2 was beneficial for initial evacuation due to the smoke-filling effect in large spaces but over time, difficulties with smoke exhaust were observed due to accumulation of the smoke layer. Scenario 3 demonstrated good smoke exhaust performance, like scenario 1 , due to forced smoke exhaust and the natural air supply from the many doors opened to the outside. However, the amount of natural air supply was not consistent, resulting in smoke stagnation in the lower levels.

In general, International Building Code 910 (IBC., 2012) suggests that a natural exhaust system should preferentially be installed in buildings with a large undivided volumes and high fire loads. Although, the performance of natural ventilation may vary depending on the temperature of the smoke (Chow and Li, 2010) in this atrium where part of the building is open vertically without fire barriers and the fire load is limited an HST confirmed that the mechanical smoke exhaust system was efficient.

To implement PBD in an atrium space, we used CFD to evaluate fire risk at the design stage and an HST to verify the performance of the smoke control equipment after the completion of construction. The objectives of PBD in this building were protecting the life of the occupants and preserving the reusability of the building. To this end, it is important to minimize the smoke exposure of occupants in the open space and to rapidly exhaust the smoke in the event of a fire.

Three scenarios were composed to analyze smoke control characteristics based on the inclusion or exclusion of mechanized or natural ventilation systems. Conducting HSTs and FDS for each scenario revealed the variables that affect airflow: forced air supply and exhaust in scenario 1, smoke exhaust window placement in scenario 2 and the natural air supply in Scenario 3. These results demonstrated the characteristics of the smoke control equipment in the target building. Further examining the effects of forced supply and exhaust on smoke control performance, we found that the smoke exhaust volume was sufficient but when the volume of the air supply was equal to or greater than the exhaust volume stipulated in related Korean standards, airflow was varied and dispersed. Second, smoke exhaust windows did not provide sufficient exhaust capacity and their positioning was inappropriate. Smoke exhaust windows are planned according to design considerations, the prioritization of indoor air circulation and limitations of installation spaces, thus, they demonstrate poor smoke exhaust performance during a fire. Third, natural air supply and exhaust through the doors connecting the atrium with the outside could provide smoke control performance. However, to use a natural air supply, it is essential to consider changes in air flow rate and flow velocity with seasonal changes, as well as the ability to automatically open doors communicating with the outside air (Hurley et al., 2015). 


\section{CONCLUSION}

In the present study, we verified the characteristic performance of smoke control equipment in different scenarios using CFD and HSTs which are common PBD methods in Korea. In the HST, heaters were used to prevent equipment damage and conduct safe experiments. Moreover, quantitative analysis of the ventilation system was conducted using the analog sensor installed in the building. These methods can be used as tools to determine the operating conditions for smoke control equipment, the effects of air supply/exhaust vent placement on smoke control and building administration measures in atria of various shapes.

\section{ACKNOWLEDGEMENTS}

We would like to thank Editage (www.editage.co.kr) for English language editing. The study did not receive any funding. The researchers have no conflicts of interest to declare.

\section{REFERENCES}

ASD., 2007. Testing and commissioning procedure for fire service installation in government buildings. Architectural Services Department, Queensway Government, Hong Kong.

Anonymous, 2015. Hot smoke testing and Australian standard AS4291. Stacey Agnew Pty Ltd, Australia.

Chow, C.L. and J. Li, 2010. An analytical model on static smoke exhaust in Atria. J. Civ. Eng. Manage., 16: 372-381.

Chow, W.K., 2009. Determination of the smoke layer interface height for hot smoke tests in big halls. J. Fire Sci., 27: 125-142.

Chow, W.K., E.C. P ang, S.S. Han, H. Dong and Y. Hou et al., 2005. Atrium hot smoke tests in a big shopping complex. J. Appl. Fire Sci., 14: 137-169.

Chow, W.K., S.S. Li, Y. Gao and C.L. Chow, 2009. Numerical studies on atrium smoke movement and control with validation by field tests. Build. Environ., 44: $1150-1155$.
Hurley, M.J., D.T. Gottuk, J.R. Hall Jr, K. Harada and E.D. Kuligowski et al., 2015. SFPE Handbook of Fire Protection Engineering. 5th Edn., Springer, Berlin, Germany, ISBN: 978-1-4939-2564-3, Pages: 3493.

IBC., 2012. 2012 International Building Code. IBC Publisher Inc, Japnies, ISBN:9781609830403, Pages: 690.

MGL., 2017. Performance-based design methods and codes for performance-based facilities. Ministry of Government Legislation, Sejong, Korea.

MLIT., 2015. Design guidelines for urban railway stations, transit and convenience facilities. Minister of Land, Infrastructure and Transport, Chiyoda, Tokyo, Japan.

McGrattan, K., S. Hostikka, R. McDermott, C. Floyd and Weinschenk et al., 2017. Fire Dynamics Simulator Technical Reference Guide. Vol. 2, Hughes Associates Inc., Baltimore, Maryland, USA.,

McGrattan, K., S. Hostikka, R. McDermott, J. Floyd and C. Weinschenk et al., 2015. Fire Dynamics Simulator User's Guide. 6th Edn., Hughes Associates Inc., Baltimore, Maryland, USA.,.

Montes, C., E. Sanmiguel-Rojas, A. Viedma and G. Rein, 2009. Experimental data and numerical modelling of 1.3 and $2.3 \mathrm{MW}$ fires in a $20 \mathrm{~m}$ cubic atrium. Build. Environ., 44: 1827-1839.

NFA., 2017. NFSC 501: National fire safety codes. Korea National Fire Agency, Korea.

NFPA., 2010a. NFPA 92, Standard for smoke control systems. National Fire Protection Association, Massachusetts, USA.

NFPA., 2010b. NFPA, 130, Standard for fixed guideway transit and passenger rail system. National Fire Protection Association, Massachusetts, USA.

NFPA., 2015. NFPA 92, Standard for smoke control systems. National Fire Protection Association, Massachusetts, USA.

Standard, A., 1999. Smoke Management Systems: Hot Smoke Test. Standards Association of Australia, Sydney, Australia, ISBN:9780733723711,

Su, C.H., Y.C. Lin, C.M. Shu and M.C. Hsu, 2011. Stack effect of smoke for an old-style apartment in Taiwan. Build. Environ., 46: 2425-2433.

Webb, A., 2006. FDS modelling of hot smoke testing, cinema and airport concourse. MS Thesis, Worcester Polytechnic Institute, Worcester, England. 